\title{
PREPARATION AND CHARACTERIZATION OF ETODOLAC BEARING EMULSOMES
}

\author{
VIVEK GILL, ARUN NANDA
}

Department of Pharmaceutical Sciences, Maharashi Dayanand University, Rohtak-124001, Haryana, India Email: gillvivek@gmail.com

Received: 23 Jun 2020, Revised and Accepted: 24 Jul 2020

\begin{abstract}
Objective: Emulsomes are novel vesicular drug delivery system with an internal solid lipid core surrounded by one or more bilayers of phospholipids. Etodolac is a potent anti-inflammatory drug and is a drug of choice for the treatment of various diseases. The present study is focused on the development of emulsomes using etodolac as drug candidates having improved drug loading with sustained-release effect for patient compliance.

Methods: Emulsomes formulation composed of solid lipids (tristearin), phospholipids, cholesterol, stearylamine, and drug (etodolac) were prepared by lipid film hydration method followed by sonication to produce emulsomes of the nanometric size range. All the formulations were optimized by using box-behnken design of experiment considering 3 factors viz. drug to phospholipid ratio (A), tristearin to phospholipid ratio (B), stearylamine to phospholipid ratio (C) at 3 levels lower $(-1)$, middle $(0)$ and upper $(+1)$. The response of the independent variables $(A, B, C)$ was studied on the dependent variable viz. particle size (Y1), zeta potential (Y2), and entrapment efficiency (Y3). The responses were analyzed by design expert software to find out the optimized values of variables within the design space.

Results: Compatibility with excipients was established by FTIR studies. The developed emulsomes were spherical shape vesicles as analyzed by TEM. The optimized batch (OB) was evaluated for particle size, zeta potential, and entrapment efficiency with experimental values $383.1 \pm 11.7$ nm, $47.2 \pm 1.3$ $\mathrm{mV}$ and $80.1 \pm 3.2 \%$ and predicted values $390.394 \mathrm{~nm}, 45.000 \mathrm{mV}$ and $81.642 \%$, respectively. The experimental values were found in reasonable agreement with predicted values by the design of the experiment. In vitro drug release study showed sustained release of the drug (88.69 \% after $24 \mathrm{~h}$ ).

Conclusion: Etodolac loaded emulsomes is a novel drug delivery system and found to reliable in terms of various characteristic parameters like particle size, zeta potential, entrapment efficiency, and drug release. 3-factors 3-levels Box-behnken design of the experiment is a suitable design for
\end{abstract} the optimization of emulsomes.

Keywords: Emulsome, Etodolac, Box-Behnken design, Phospholipid, Tristearin

(C) 2020 The Authors. Published by Innovare Academic Sciences Pvt Ltd. This is an open access article under the CC BY license (http://creativecommons.org/licenses/by/4.0/) DOI: http://dx.doi.org/10.22159/ijap.2020v12i5.38842. Journal homepage: https://innovareacademics.in/journals/index.php/ijap

\section{INTRODUCTION}

Lipid-based drug delivery systems have gained the attention of researchers in the recent few decades due to their certain advantages over other drug delivery systems. Out of various lipidbased drug delivery system viz. liposomes, solid lipid nanoparticles, niosomes, ethosomes, emulsomes, glycerosomes, etc $[1,2]$. Emulsomes have been proven to be advantageous for the delivery of lipophillic drugs. Emulsomes is a lipid-based, vesicular drug delivery carrier having structurally similarity with liposomes but differs only in core component as emulsomes unlike liposomes is composed of solid core [3]. Solid core is composed up of solid lipid which remains solid at $25{ }^{\circ} \mathrm{C}$ and having solid to liquid phase transition temperature near to physiological temperature $[4,5]$. Due to lipid core, this system becomes advantageous as a carrier system for lipophillic drugs having high drug entrapped by encapsulating lipophillic drugs in lipid core as well as between phospholipid bilayers [6]. Drug entrapped in solid lipid core also exhibits sustained release [7]. Charge inducers are also added in emulsomes to induce surface charge to the vesicles to prevent aggregation of vesicles having a similar charge and stabilize the formulation by providing mono-dispersed vesicles [8]. Emulsomes can be used for the delivery of drugs via oral, parenteral, rectal, topical, intranasal or ocular [9]. Therefore emulsomes may be considered as an efficient drug delivery system because of biocompatibility, biodegradability, stability in the gastrointestinal tract, high entrapment efficiency, and sustained drug release [10,11].

Etodolac is a non-steroidal anti-inflammatory drug that is used in the treatment of rheumatoid arthritis that belongs to pyranocarboxylic acid group [12]. Etodolac is a selective COX-2 inhibitor, which is an enzyme responsible for the regulation of prostaglandins (inflammatory mediators) [13]. Etodolac is a white crystalline powder insoluble in water and soluble in alcohols, chloroform, dimethylsulfoxide, and polyethylene glycol. It is a very lipophillic drug and exists as a racemic mixture of $(+) S$ and $(-)$ R enantiomer. Despite its various applications, etodolac causes gastrointestinal disturbances, including peptic ulcers and gastrointestinal bleeding, due to these problems oral use of etodolac is avoided [14].

Response surface type designs of experiments have been widely used for the optimization of various process parameters for vesicular drug delivery systems [15]. The advantage of response surface type designs of experiments is that despite using a single factor, multiple factors can be studied at a time, including their interaction effect [16]. Various types of design using response surface methodology are doehlert matrix (DM), box-behnken design (BBD), and central composite design (CCD) [17]. Out of this box-behnken design has been mainly used to study the effect of 3 factors at 3 levels $[18,19]$. In this design quadratic equation is generated for evaluating the mathematical relationship between independent and dependent variables to study the effect of the independent variable on the independent variable [20]. 3dimensional response curves and 2-dimensional contour plots help in studying the response of 2 factors, including their interaction effect keeping other factors constant $[17,21]$

\section{MATERIALS AND METHODS}

Material

Etodolac was procured from Balaji chemicals Surat, tristearin, and lecithin were procured from HiMedia, cholesterol was procured from LOBA chemie Mumbai, sephadex G-50 was procured from Sigma aldrich USA, stearylamine was procured from Ottokemi, Mumbai. All the other solvents and chemicals used were of analytical grade.

\section{Method of preparation of emulsomes}

Emulsomes encapsulating etodolac were prepared by the lipid film hydration method as described by Gupta and Vyas [10] with slight modifications as per laboratory set up. To a $500 \mathrm{ml}$ round bottom flask 
(RBF) accurately weighed amount of lecithin, tristearin, cholesterol and stearylamine were dissolved in a small amount of chloroform. In a separate beaker, etodolac was dissolved in methanol. Drug solution in methanol was transferred into RBF having other ingredients dissolved in chloroform. Both the solutions were mixed and the organic solvent was evaporated until complete dryness under reduced pressure using a rotary evaporator. Thin dry film was formed over the inner wall of RBF. Dry film was hydrated using phosphate buffer saline ( $\mathrm{pH}$ 7.4). Hydration of dry film facilitates swelling of lipids and formation of emulsomes vesicles dispersed in the aqueous phase. The solution was sonicated by probe sonicator to obtain nano-sized emulsomes vesicles. The free un-entrapped drug was removed by passing through sephadex G-50 column [22].

\section{Optimization of emulsomes formulation}

The formulation was optimized using a box-behnken design of experiment, which is a response surface type design of experiment wherein responses of 3 factors were studied at 3 levels. Three factors considered were phospholipid to etodolac ratio (A); phospholipid to tristearin ratio (B) and phospholipid to stearyl amine ratio $(C)$ at three levels upper, middle and lower level $(+1,0$, 1). A total no. of 17 experiments were designed with 5 center points and 12 points at edges of design space for estimation of pure error sum of squares to choose the best model among linear, two-factor interaction and quadratic model due to the analysis of variance (ANOVA), F-value [23]. The effect of independent variables $(A, B, C)$ were studied on dependent variables i.e. particle size (Y1), zeta potential (Y2) and entrapment efficiency (Y3) by constructing their response surface models along with quadratic equation using design expert software (Design-expert software, version 11, State-Ease Inc. Minneapolis, MN). The designed quadratic polynomial equation generated is as follows:

$$
\begin{gathered}
Y_{o}=b_{o}+b 1 X 1+b 2 X 2+b 3 X 3+b 12 X 1 X 2+b 13 X 1 X 3+b 23 X 2 X 3 \\
+b 11 X 1^{2}+b 22 X 2^{2}+b 33 X 3^{2}
\end{gathered}
$$

Where ${ }_{0}$ is a response for each dependent variable; $b_{\circ}$ is an intercept; b1,b2,b3,b12,b13,b23,b11,b22,b33 are regressed coefficients from experimental response values of $\mathrm{Y}$; X1, X2, X3 and their combinations $(\mathrm{X} 1 \mathrm{X} 2, \mathrm{X} 1 \mathrm{X} 3, \mathrm{X} 2 \mathrm{X} 3)$ and square values $\left(\mathrm{X}^{2}, \mathrm{X}^{2}\right.$, $\mathrm{X}^{2}$ ) represented terms for studying the interactive effect of two factors on the response at the same time simultaneously and to evaluate the fitness of the model, predicted $R^{2}$ and adjusted $R^{2}$. The values of independent variables $(A, B, C)$ and the constraints of dependent variables (Y1, Y2, Y3) are shown in table 1.

Table 1: Variables used in the box-behnken design of experiment

\begin{tabular}{lll}
\hline Independent variables & Levels & $\mathbf{0}$ \\
\cline { 2 - 3 } & $\mathbf{- 1}$ & $\mathbf{+ 1}$ \\
\hline A $=$ Etodolac to Phospholipid ratio (\% w/w of Phospholipid) & $1 \%$ & $3 \%$ \\
B $=$ Tristearin to Phospholipid ratio (\% w/w of Phospholipid) & $50 \%$ & $100 \%$ \\
C $=$ Stearylamine to Phospholipid ratio (\% w/w of Phospholipid) & $5 \%$ & $10 \%$ \\
Dependent Variables & Constraints & \\
Y1 $=$ Particle Size (nm) & Minimum & \\
Y2 $=$ Zeta Potential (mV) & Maximum & \\
Y3 $=$ Entrapment efficiency (\%) & Maximum & \\
\hline
\end{tabular}

\section{Drug and excipients compatibility studies}

Etodolac and other ingredients i.e. lecithin, tristearin, stearyl amine, and cholesterol were mixed separately in the ratio of $1: 1$ and all ingredients, including drug, were also mixed in equal proportions to form a physical mixture. The mixtures were placed in properly sealed glass vials and vials were kept at room temperature. The FTIR spectra of drug, excipients, and their physical mixtures were recorded and analyzed for any deviation in principle peaks of etodolac. The spectra of all the samples were analyzed at $4000-600 \mathrm{~cm}^{-1}$.

\section{Characterization of optimized emulsomes}

\section{Transmission electron microscopy}

The emulsomes were characterized for their shape and surface morphology by using a transmission electron microscope (TEM)(Hitachi 7500, Japan). Phosphotungstic acid (1\%) was used as a negative stain. Carbon coated samples were placed over a copper grid and subjected to TEM analysis.

\section{Particle size and zeta potential measurement}

Emulsomes samples were analyzed in triplicate in an aqueous medium. Average particle size and zeta potential were measured at $25^{\circ} \mathrm{C}$ by zeta sizer (PCS; Zetasizer, HAS 3000; Malvern Instruments, Malvern, UK). All the measurements were carried out with an angle of $90^{\circ}$ at $25^{\circ} \mathrm{C}$ [24].

\section{Determination of drug entrapment efficiency}

Emulsomes dispersion was drop-wise filtered through sephadex G50 column. Filterate was treated with a few drops of triton X-100. Triton X-100 breaks the phospholipid bilayer of emulsomes vesicles and the entrapped drug comes out in solution, which was analyzed by HPLC technique to determine the area under the curve for evaluation of entrapped drug [24]. Drug entrapment efficiency was calculated using the formula:

Entrapment efficiency (\%)

$$
=\frac{\text { Amount of drug encapsulated in vesicles }}{\text { Initial amount of drug taken }} \times 100
$$

\section{In vitro drug release study}

In vitro dissolution studies were performed by the dialysis membrane sac technique with the cellophane membrane of molecular weight $12000 \mathrm{D}$ [26]. The formulation was placed in a dialysis membrane bag and was placed in a vessel having a dissolution medium $(100 \mathrm{ml})$. The dissolution medium was maintained at a physiological temperature of $37 \pm 1{ }^{\circ} \mathrm{C}$ with constant stirring with a magnetic bead at $50 \mathrm{rpm}$ on the magnetic stirrer (REMI, India) [27]. The dialysis membrane sac was hanged in a medium. At predetermined intervals $(0.5,1,2,4,6,8,10,12,24 \mathrm{~h})$ the aliquots of $1 \mathrm{ml}$ were taken sink conditions were maintained by replacing the equal volume of fresh medium. Samples were analyzed for the amount of etodolac released with HPLC method.

\section{RESULTS AND DISCUSSION}

\section{Drug-excipients compatibility studies}

The drug-excipients compatibility studies were performed by analyzing FTIR spectrum of samples for any deviation in principle peaks of the drug in the spectrum. As shown in fig. 1 no deviation of principle peaks was observed in spectra of etodolac when compared with the various spectrum of physical mixtures. Results confirm the physical compatibility of the drug with the excipients used in the formulation.

\section{Preparation and optimization by the box-behnken design of} experiment

Etodolac loaded emulsomes were prepared by the lipid film hydration method. Based on preliminary studies and experimental trial runs, three levels of each independent variable were decided for studies. Box-behnken design of the experiment was considered to be the best suitable design of the experiment for studying the effect of three variables at three levels. On applying the design of the experiment using design expert software total of 17 runs with 5 center points and 12 edge points within the design space were obtained [28]. All the 17 batches were prepared and their responses were recorded as shown in table 2. It was observed from the responses obtained that independent variables $(A, B, C)$ have a 
significant effect on the dependent variables (particle size, zeta potential, entrapment efficiency). Responses obtained from 17 experimental runs were put in design expert software to obtain results fitted to first-order, second-order, and quadratic models along with predicted values and conclusion. Responses were analyzed for the best fit model with significant quadratic $(\mathrm{p}<0.0001)$ and insignificant lack of fit $(\mathrm{p}>0.0525)$ as shown in table 3. Three dimensional (3-D) response curves were generated as shown in fig.
2. Response curves showed the interaction effect independent variables on the dependent variable and useful in studying the effect of two factors on one response at a time [15]. Polynomial equations were also generated for all three responses (Y1, Y2, Y3). Equations helped evaluate the effect of an individual as well as the interaction of variables on the responses [17]. A positive sign in the equation for a factor represents a synergistic effect on the response, while a negative sign represents the antagonistic effect.

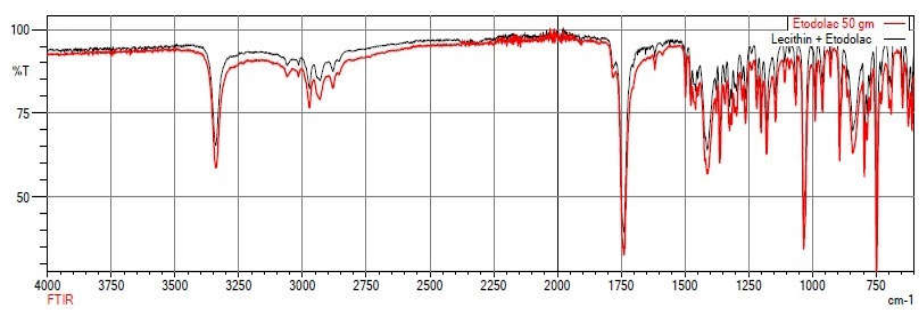

(A)

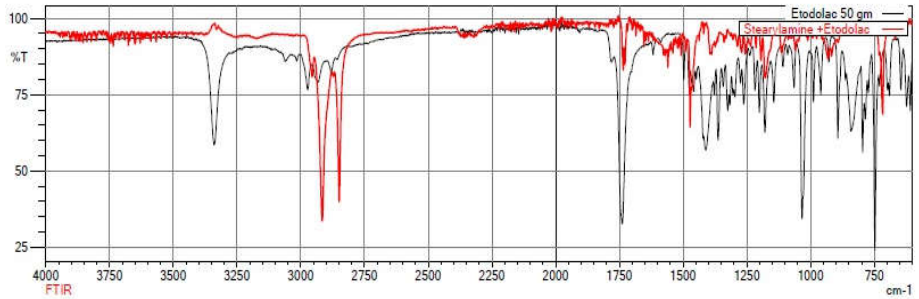

(B)

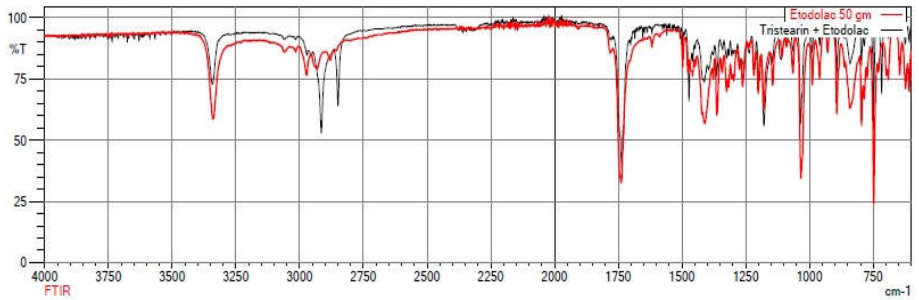

(C)

Fig. 1: Compatibility studies by analysing FTIR spectrum of various samples. (A) Comparison of spectra of etodolac with physical mixture (lecithin+etodolac), (B) Comparison of spectra of etodolac with physical mixture (stearylamine+etodolac), (C) Comparison of spectra of etodolac with physical mixture (tristearin+etodolac)

Table 2: Experimental layout for 3 factors 3 levels box-behnken design and values of response variables

\begin{tabular}{|c|c|c|c|c|c|c|}
\hline \multirow[t]{3}{*}{ Batches } & \multicolumn{3}{|l|}{ Variables } & \multirow{2}{*}{\multicolumn{3}{|c|}{ Responses }} \\
\hline & A & B & $\mathrm{C}$ & & & \\
\hline & Drug: PHL (in mg) & TRI: PHL (in mg) & STR: PHL (in mg) & Size (nm) & Zeta potential (mV) & $\begin{array}{l}\text { Entrapment efficiency (\% } \\
\text { age) }\end{array}$ \\
\hline F1 & 1 & 50 & 10 & 206 & 56.4 & 69.42 \\
\hline F2 & 5 & 50 & 10 & 405 & 57.4 & 76.46 \\
\hline F3 & 1 & 150 & 10 & 564 & 52.1 & 78.67 \\
\hline $\mathrm{F} 4$ & 5 & 150 & 10 & 641 & 55.8 & 88.46 \\
\hline F5 & 1 & 100 & 5 & 432 & 27.8 & 74.82 \\
\hline F6 & 5 & 100 & 5 & 488 & 28.3 & 81.67 \\
\hline F7 & 1 & 100 & 15 & 651 & 66.8 & 73.41 \\
\hline F8 & 5 & 100 & 15 & 780 & 59.3 & 83.88 \\
\hline F9 & 3 & 50 & 5 & 189 & 22.6 & 69.68 \\
\hline F10 & 3 & 150 & 5 & 492 & 25.7 & 75.46 \\
\hline F11 & 3 & 50 & 15 & 489 & 68.5 & 72.78 \\
\hline F12 & 3 & 150 & 15 & 678 & 64.4 & 77.67 \\
\hline F13 & 3 & 100 & 10 & 378 & 42.3 & 80.56 \\
\hline F14 & 3 & 100 & 10 & 392 & 45.5 & 82.02 \\
\hline F15 & 3 & 100 & 10 & 365 & 44.1 & 79.96 \\
\hline F16 & 3 & 100 & 10 & 388 & 48.7 & 81.78 \\
\hline F17 & 3 & 100 & 10 & 390 & 44.8 & 79.41 \\
\hline
\end{tabular}


Table 3: Analysis of variance (ANOVA) of the calculated model for responses

\begin{tabular}{llll}
\hline Result of the ANOVA & Particle size (nm) & Zeta potential (mV) & Entrapment efficiency (\%) \\
\hline Regression: & & 3339.59 & 358.02 \\
Sum of Squares & $4.013 \mathrm{E}+05$ & 9 & 9 \\
Degree of freedom (df) & 9 & 371.07 & 39.78 \\
Mean square & 44583.44 & 37.75 & 26.16 \\
F-value & 106.99 & $<0.0001$ & $<0.0001$ \\
p-value & $<0.0001$ & Significant & Significant \\
Inference & Significant & & 5.51 \\
Lack of fit tests: & 2413.75 & 46.76 & 3 \\
Sum of squares & 3 & 3 & 1.84 \\
Degree of freedom (df) & 804.58 & 15.59 & 1.43 \\
Mean square & 6.40 & 2.83 & 0.3575 \\
F-value & 0.0525 & 0.1705 & Not significant \\
p-value & Not significant & Not significant & \\
Inference & & & 10.64 \\
Residual: & 2916.95 & 68.81 & 7 \\
Sum of Squares & 7 & 7 & 1.52 \\
Degree of freedom (df) & 416.71 & 9.83 & \\
Mean square & & & \\
\hline
\end{tabular}

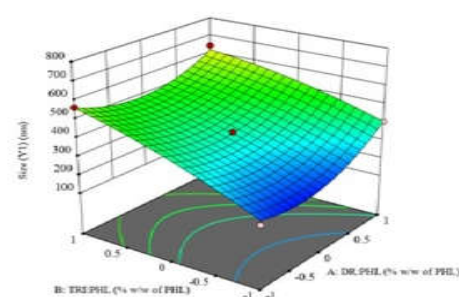

(A1)

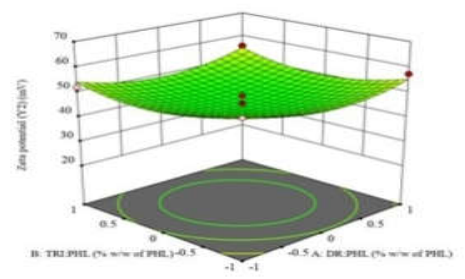

(B1)

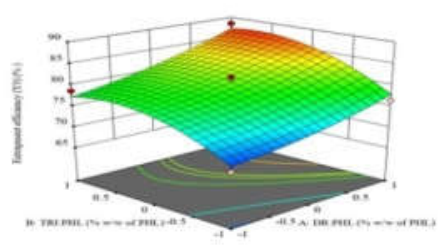

(C1)

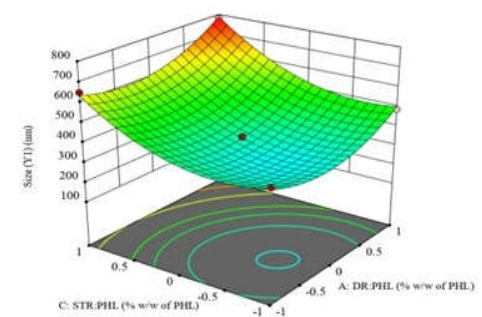

(A2)

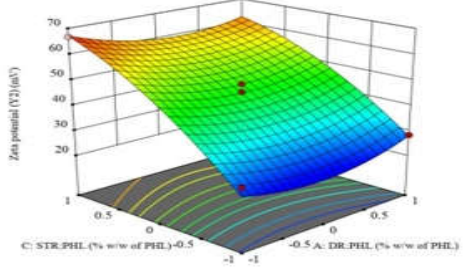

(B2)

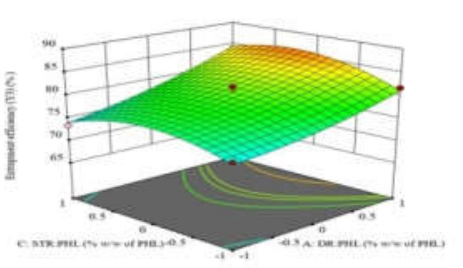

(C2)

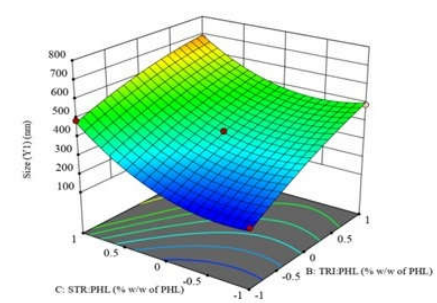

(A3)

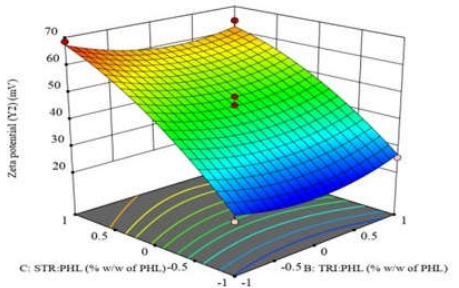

(B3)

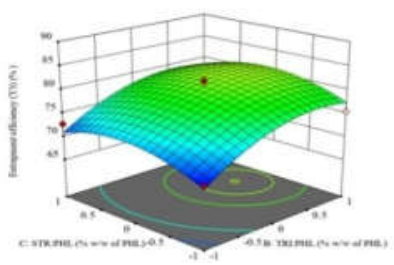

(C3)

Fig. 2: 3-dimensional response curves showing: (A1) Effect of factors A and B on particle size; (A2) Effect of factors A and C on particle size; (A3) Effect of factors B and C on particle size; (B1) Effect of factors A and B on zeta potential; (B2) Effect of factors A and C on zeta potential; (B3) Effect of factors B and C on zeta potential; (C1) Effect of factors A and B on entrapment efficiency; (C2) Effect of factors A and $C$ on entrapment efficiency; (C3) Effect of factors $B$ and $C$ on entrapment efficiency

The polynomial equation of response $\mathrm{Y} 1$ (particle size) is shown as: $\mathrm{Y} 1=382.60+57.63 \mathrm{~A}+135.75 \mathrm{~B}+124.63 \mathrm{C}-30.50 \mathrm{AB}+18.25 \mathrm{AC}$ $-28.50 \mathrm{BC}+98.58 \mathrm{~A}^{2}-27.18 \mathrm{~B}^{2}+106.58 \mathrm{C}^{2}$

The model $F$ value of 106.99 for particle size implied that the model was significant $(\mathrm{p}<0.0001)$ with a lack of fit value of 6.40 , which was not significant $(\mathrm{p}=0.0525)$ [29]. As shown in polynomial equation factors A, B, C and interaction of AC have a synergistic effect, while the interaction of $\mathrm{AB}$ and $\mathrm{BC}$ has an antagonistic effect on particle size. The predicted $\mathrm{R}^{2}$ value of 0.9025 is justified with an adjusted $\mathrm{R}^{2}$ value of 0.9835 , which indicates the adequacy of the model to predict the response of particle size. Adeq. precision measures the signal to noise ratio and a ratio greater than 4 is desirable. Adeq. precision value 39.289 indicated an adequate signal [30].

The polynomial equation of response Y2 (zeta potential) is shown as:

$\mathrm{Y} 2=45.08-0.2875 \mathrm{~A}-0.8625 \mathrm{~B}+19.33 \mathrm{C}+0.6750 \mathrm{AB}-2.00 \mathrm{AC}$ $-1.80 \mathrm{BC}+5.30 \mathrm{~A}^{2}+5.05 \mathrm{~B}^{2}-4.83 \mathrm{C}^{2}$ 
The model $\mathrm{F}$ value of 37.75 for zeta potential implied that the model was significant $(\mathrm{p}<0.0001)$ with a lack of fit value of 2.83 , which was not significant ( $p=0.1705)$ [29]. As shown in polynomial equation factors $C$ and interaction of $A B$ have a synergistic effect, while factors $\mathrm{A}, \mathrm{B}$ and interactions of $\mathrm{AC}$ and $\mathrm{BC}$ have an antagonistic effect on zeta potential. The predicted $\mathrm{R}^{2}$ value of 0.7704 is justified with an adjusted $\mathrm{R}^{2}$ value of 0.9539 , which indicates the adequacy of the model to predict the response of zeta potential. Adeq. precision measures the signal to noise ratio and a ratio greater than 4 is desirable. Adeq precision value 17.7890 indicated an adequate signal [30]

The polynomial equation of response Y3 (entrapment efficiency) is shown as:

$$
\begin{aligned}
\mathrm{Y} 3=80.75+4.40 \mathrm{~A} & +3.27 \mathrm{~B}+0.7637 \mathrm{C}-0.7475 \mathrm{AB}+0.9050 \mathrm{AC} \\
& -0.2225 \mathrm{BC}+1.162 \mathrm{~A}^{2}-3.385 \mathrm{~B}^{2}-3.463 \mathrm{C}^{2}
\end{aligned}
$$

The model $\mathrm{F}$ value of 26.16 for entrapment efficiency implied that the model was significant $(\mathrm{p}<0.0001)$ with a lack of fit value of 1.43 , which was not significant $(p=0.3575)$ [29]. As shown in polynomial equation factors $\mathrm{A}, \mathrm{B}, \mathrm{C}$ and interaction of $\mathrm{AC}$ have a synergistic effect, while the interaction of $\mathrm{AB}$ and $\mathrm{BC}$ has an antagonistic effect on entrapment efficiency. The predicted $R^{2}$ value of 0.7389 is justified with an adjusted $\mathrm{R}^{2}$ value of 0.9340 , which indicates the adequacy of the model to predict the response of entrapment efficiency. Adeq. precision measures the signal to noise ratio and a ratio greater than 4 is desirable. Adeq. precision value 16.7196 indicated an adequate signal [30]. All the values of various parameters of the design about to with concerning responses were justified as per design. Therefore this model was used to navigate the design space.

\section{Optimization}

The effects of various variables were studies on the responses and the responses were analyzed for different parameters as discussed above. Finally, the optimized values of all the three variables considered in the study were evaluated by the desirability criteria [31]. Desirability criteria in emulsomes formulation were minimum particle size with maximum zeta potential and entrapment efficiency. The maximum value of desirability is 1 and the values of variables having desirability fig. near to 1 are considered to be optimized [31] for formulating emulsomes. As evaluated by design expert software, the optimized values within design space having maximum desirability of 0.797 are selected for formulation shown in table 4 .

Table 4: Optimized values of variables

\begin{tabular}{lllllll}
\hline Desirability & \multicolumn{2}{l}{ Optimized value of variables } & \multicolumn{3}{l}{ Predicted response } \\
\cline { 2 - 7 } & $\begin{array}{l}\text { DRUG: PHL (\% } \\
\text { w/w of PHL) }\end{array}$ & $\begin{array}{l}\text { TRI: PHL (\% } \\
\text { w/w of PHL) }\end{array}$ & $\begin{array}{l}\text { STR: PHL (\% } \\
\text { w/w of PHL) }\end{array}$ & $\begin{array}{l}\text { Particle size } \\
\text { (nm) }\end{array}$ & $\begin{array}{l}\text { Zeta potential } \\
\text { (mV) }\end{array}$ & $\begin{array}{l}\text { Entrapment } \\
\text { efficiency (\%) }\end{array}$ \\
\hline 0.797 & 3.834 & 90.171 & 9.689 & 390.394 & 45.000 & 81.642 \\
\hline
\end{tabular}

\section{Surface morphology}

The prepared emulsomes were examined under a transmission electron microscope (TEM) for surface morphological studies. The image showed that the emulsomes were spherical [9]. All the emulsomes vesicles were observed to be mono-dispersed and no sign of aggregated vesicles was observed. The TEM image is shown in fig. 3.

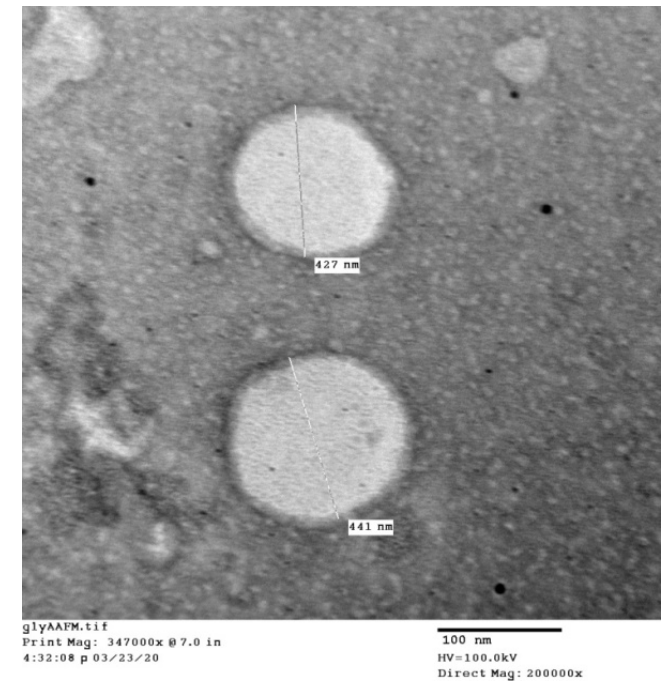

Fig. 3: TEM image of optimized batch (OB)

\section{Particle size and zeta potential}

The average particle size and zeta potential of various batches vary from $189 \mathrm{~nm}$ to $780 \mathrm{~nm}$ and $22.6 \mathrm{mV}$ to $68.5 \mathrm{mV}$, respectively. The average particle size and zeta potential of the optimized batch were found to be $383.1 \pm 11.7 \mathrm{~nm}$ and $47.2 \pm 1.3 \mathrm{mV}$, respectively (fig. 4 and fig. 5 ).

\section{Drug entrapment efficiency}

The samples were analyzed by HPLC method, and the area of chromatogram was analyzed to determine the amount of drug present in the sample. The drug entrapment efficiency of emulsomes batches varies from $69.42 \%$ to $88.46 \%$ and the entrapment efficiency of the optimized batch was found to be $80.1 \pm 3.2 \%$. The high entrapment efficiency was observed in emulsomes as compared to other vesicular drug delivery carriers. The lipophillic nature of etodolac enhances the high amount of drug to be incorporated in between the phospholipid bilayer as well as in the solid lipid core of emulsomes $[7,10]$.

Thus, more entrapment efficiency was observed. 


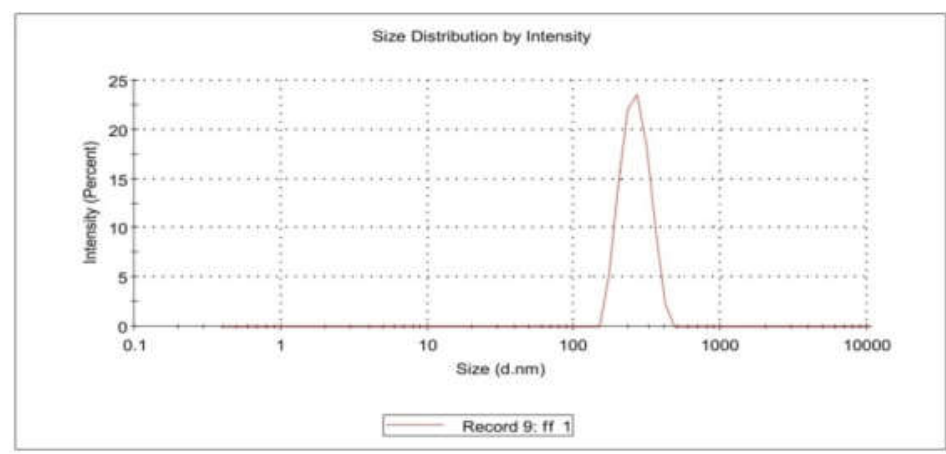

Fig. 4: Particle size report

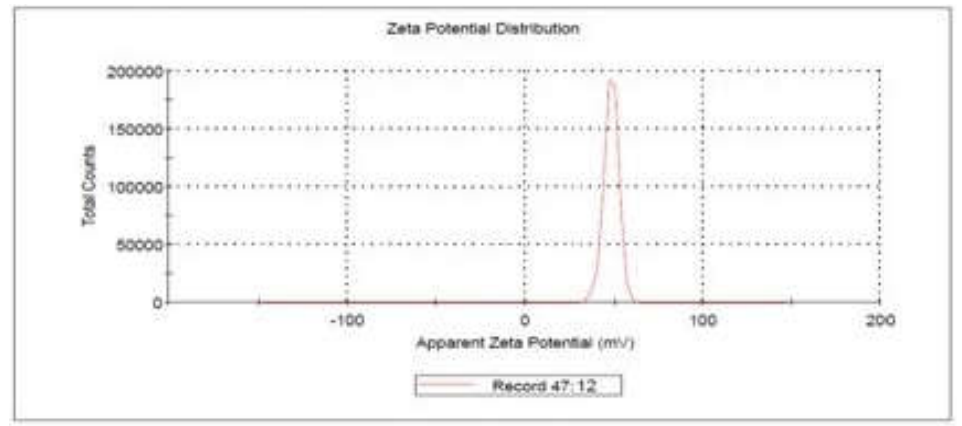

Fig. 5: Zeta potential report

\section{In vitro drug release study}

The in vitro drug release study of an optimized batch was carried out using a dialysis membrane. The dialysis membrane allows drug molecules to diffuse through but restricts the diffusion of emulsomes vesicles [8]. The cumulative drug release during $1^{\text {st }}$ hour of $28.45 \%$ indicated the initial burst release, which may be due to the initial release of drug from the phospholipid bilayer. After $1 \mathrm{~h}$ the release pattern showed slow release of the drug over $24 \mathrm{~h}$ study up to 88.69 $\%$. The sustained release of drug is due to the slow release of drug entrapped in solid lipid core composed of tristearin [25]. The overall release study confirms the release of drug in a controlled manner up to $24 \mathrm{~h}$ with a cumulative drug release of $88.69 \%$. The cumulative drug release profile of the optimized batch is shown in fig. 6 .

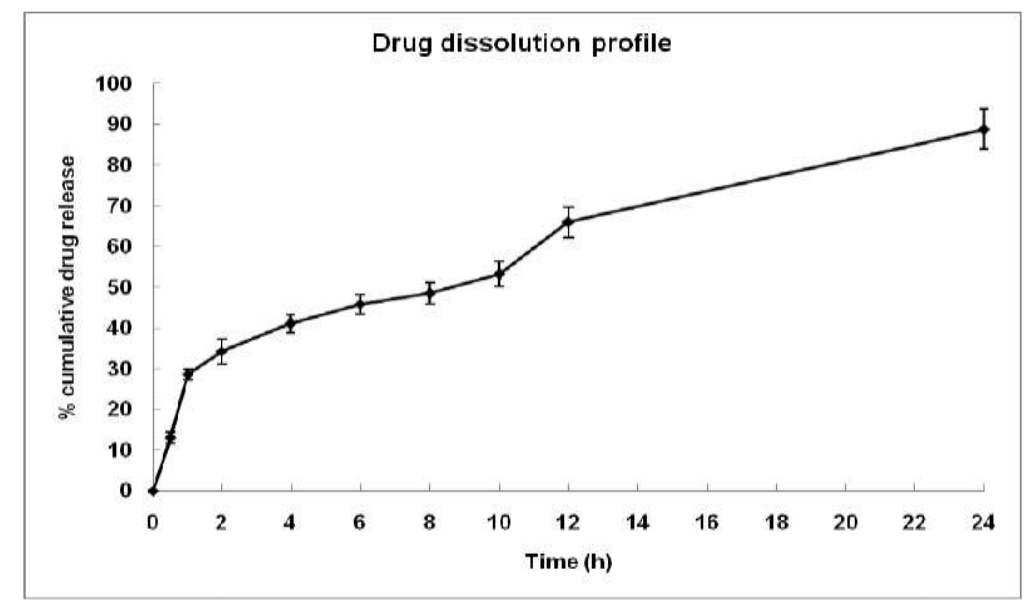

Fig. 6: In vitro drug release profile of optimized batch $(\mathrm{OB}),{ }^{*}$ values are shown in $\mathrm{mean} \pm \mathrm{SD}(\mathrm{n}=3)$

\section{CONCLUSION}

Etodolac loaded emulsomes were successfully prepared by the lipid film hydration method. The formulation was optimized by using a box-behnken design of experiment and results revealed that boxbehnken design is a suitable design of experiment for optimizing formulation by considering 3 factors at 3 levels. Results of various parameters i.e. particle size $(383.1 \pm 11.7 \mathrm{~nm})$, zeta potential $(47.2 \pm 1.3 \mathrm{mV})$ and entrapment efficiency $(80.1 \pm 3.2 \%)$ showed reasonable agreement with predicted values by the design of experiment as particle size $(390.394 \mathrm{~nm})$, zeta potential $(45.000$ $\mathrm{mV})$ and entrapment efficiency (81.642\%). The difference in 
experimental values and predicted values are not significant, which proves the suitability of the box-behnken design of experiment with formulation for optimization. In vitro drug release study showed sustained release of the drug throughout for $24 \mathrm{~h}$. Thus it can be concluded that emulsomes are promising drug delivery system for lipophillic drugs like etodolac with better entrapment efficiency and sustained drug release, which can be optimized by box-behnken design of experiment.

\section{ACKNOWLEDGMENT}

The authors are thankful to Director, Aryabhatta Central Instrumentation Laboratory, Maharashi Dayanand University, Rohtak, Haryana, India for allowing to use instrumentation facilities.

\section{FUNDING}

No funding was availed in the present work.

\section{AUTHORS CONTRIBUTIONS}

All the authors have contributed equally.

\section{CONFLICT OF INTERESTS}

The authors report no conflict of interest.

\section{REFERENCES}

1. Jain KK. Drug delivery systems-an overview. In: Jain KK. (ed.) Drug Delivery Systems. Totowa: Humana Press; 2008. p. 1-50.

2. Lala RR, Shinde AS, Nandvikar NY. Solid lipid nanoparticles: a promising approach for combinational drug therapy in cancer. Int J Appl Pharm 2018;10:17-22.

3. Lowell GH, Kaminski RW, Van Cott TC, Slike B, Kersey K Zawoznik E, et al. Proteosomes, emulsomes, and cholera toxin B improve nasal immunogenicity of human immunodeficiency virus gp160 in mice: induction of serum, intestinal, vaginal, and lung IgA and IgG. J Infect Dis 1997;175:292-301.

4. Amselem AS, Yogev A, Zawoznik E, Friedman D. In emulsomes, a novel drug delivery technology. International Symposium on Control and Release of Bioactive Materials; 1994. p. 1369.

5. Amselem AS, Friedman D. Solid fat nanoemulsion, United States Patent no. 5,662,932(02-09-1997); 1997.

6. Kretschmar M, Amselem S, Zawoznik E, Mosbach K, Dietz A, Hof $\mathrm{H}$, et al. Efficient treatment of murine systemic infection with Candida albicans using amphotericin B incorporated in nanosize range particles (emulsomes). Mycoses 2001;44:281-6.

7. Paliwal R, Paliwal SR, Mishra N, Mehta A, Vyas SP. Engineered chylomicron mimicking carrier emulsomes for lymph targeted oral delivery of methotrexate. Int J Pharm 2009;380:181-8.

8. Vyas SP, Subhedar R, Jain S. Development and characterization of emulsomes for sustained and targeted delivery of an antiviral agent to liver. J Pharm Pharmacol 2006;58:321-6.

9. Ucisik MH, Sleytr UB, Schuster B. Emulsomes meet S-layer proteins: an emerging targeted drug delivery system. Curr Pharm Biotechnol 2015;4:392-405.

10. Gupta S, Vyas SP. Development and characterization of Amphotericin B bearing emulsomes for passive and active macrophage targeting. J Drug Target 2007;15:206-17.

11. Ucisik MH, Kupcu S, Schuster B, Sleytr UV. Characterization of curcu emulsomes: nanoformulation for enhances solubility and delivery of curcumin. J Nanobiotechnol 2013;11:37.

12. Jones RA. Etodolac: an overview of a selective COX-2 inhibitor Inflammopharmacology 1999;7:269-75.

13. Colebatch AN, Marks JL, Edwards CJ. Safety of non-steroidal anti-inflammatory drugs, including aspirin and paracetamol (acetaminophen) in people receiving methotrexate for inflammatory arthritis (rheumatoid arthritis, ankylosing spondylitis, psoriatic arthritis, other spondyloarthritis). Cochrane Database Syst Rev 2011;11:CD008872.

14. James E, Reynolds F. Martindale: the extra pharmacopoeia. London: Royal Pharmaceutical Society; 1996.

15. Singh B, Dahiya M, Saharan V, Ahuja N. Optimizing drug delivery system using systemic "Design of experiments" part-2 fundamental aspects. Crit Rev Ther Drug 2005;2:215-93.

16. El-Malah Y, Nazzal S, Khanfar N. D-optimal mixture design: optimization of ternary matrix blends for controlled zero order drug release from oral dosage forms. Drug Dev Ind Pharm 2006;32:1207-18.

17. Singh B, Kumar R, Ahuja N. Optimizing drug delivery system using systemic "Design of experiments" part-1 fundamental aspects. Crit Rev Ther Drug 2004;22:27-105.

18. Moghddam SMM, Ahad A, Aquil M, Imam SS, Sultana Y. Optimization of nanostructured lipid carriers for topical delivery of nimesulide using box-behnken design approach. Artif Cells Nanomed Biotechnol 2017;45:617-24.

19. Rane S, Prabhakar B. Optimization of paclitaxel containing $\mathrm{pH}$ sensitive liposomes by 3-factor, 3-level box-behnken design. Indian J Pharm Sci 2013;75:420-6.

20. Rathee S, Kamboj A. Optimization and development antidiabetic phytosomes by box-behnken design. J Liposome Res 2017;28:1-26.

21. Kraisit P, Sarisuta N. Development of triamcinolone acetanide loaded nanostructured lipid carriers (NLCs) for buccal drug delivery using box-behnken design. Molecules 2018;23:982.

22. New RRC. Introduction and preparation of liposomes. In: New RRC. ed. Liposomes: A practical approach. Oxford: IRL Press; 1990. p. 1-104.

23. Govinder S, Pillay V, Chetty DJ. Optimization and characterization of bioadhesive controlled release tetracycline microspheres. Int J Pharm 2005;306:24-40.

24. Jores K, Mehnert W, Drechsler M, Bunjes H, Johann C, Mader K. Investigations on the structure of solid lipid nanoparticles by photon correlation spectroscopy, field flow fractionation and transmission electron microscopy. J Controlled Release 2004;95:217-27.

25. Pal A, Gupta S, Jaiswal A, Dube A, Vyas SP. Development and evaluation of tripalmitin emulsomes for treatment of experimental visceral leishmanisis. J Liposome Res 2012;22:62-71.

26. Singh N, Verma PK, Nanda S. Nanotechnology based oral formulations of tolbutamide by using biodegradable polymer. Int J Pharm Sci 2019;10:5599-605.

27. Shen J, Burgess DJ. In vitro dissolution testing strategies for nanoparticulate drug delivery systems: recent developments and challenges. Drug Delivery Transl Res 2013;3:409-15.

28. Yasir M, Sara UVS, Chauhan I, Gaur PK, Singh AP, Puri D. Solid lipid nanoparticles for nose to brain delivery of donepezil: formulation, optimization by box-behnken design, in vitro and in vivo evaluation. Artif Cells Nanomed Biotechnol 2018;46:1838-51.

29. Dholakia M, Dave R, Thakkar V, Rana H, Gohel M, Patel N. Newer ophthalmic in situ gel of moxifloxacin hydrochloride: optimization using box behnken stastical design. Int J Pharm Pharm Sci 2018;10:5-13.

30. Gopi G, Kannan K. Formulation development and optimization of nateglinide loaded ethyl cellulose nanoparticles by boxbehnken design. Int J Pharm Pharm Sci 2015;7:310-5.

31. Ferreira SLC, Bruns RE, Ferreira HS, Matos GD, David JM, Brandao GC, et al. Box-Behnken design: an alternative for the optimization of analytical methods. Anal Chim Acta 2007;597:179-86. 